\title{
Numerical Simulation of Orientation Distribution of Fiber Suspensions in Shear-Equibiaxial Extensional Flow
}

\author{
Zan Huang \\ School of Marine engineering \\ Guangzhou Maritime Institute \\ Guangzhou 510725, China \\ E-mail: zhuanggz@aliyun.com
}

\begin{abstract}
In this paper, a mathematical model is established to apply for orientation distribution of short fibers suspensions in shear-equibiaxial extensional flow. Furthermore, the result of differential equation on plant fibers orientation can be acquired in complex flow.
\end{abstract}

Keywords-numerical simulation; orientation distribution; dynamics analysis; shear-equibiaxial extensional flow

\section{INTRODUCTION}

In recent years, the orientation distribution and behavior of fibers in fiber suspension is a hot issue in many science and eigineering fields, such as extrusion, injection, and compression molding. The reason is the orientation distribution of fibers determines largely the mechanical properties of polymer-fiber composites. Namely, the final product has stiffer and stronger properties in the direction of greatest orientation; however, it has weaker and more compliant properties in the direction of least orientation.

There has existed some literature dealing with the evolution of fiber orientation by experimental, numerical and analytical methods for decades. Parsheh et al. [1] investigated the influence of shape of planar contractions on the orientation distribution of stiff fibers suspended in turbulent flow. Tucker et al [2-3] investigated orientation fiber proportion of general fiber along with the given orientation direction in the given small research space. Lin et al. [4] obtained fiber orientation distribution in round turbulent jet of fiber suspension by numerical simulation methods. Najam ul Qadir, David A. Jack [5] used a novel methodology in formulating a closure by employing an artificial neural network (ANN) to obtain model of fiber orientation in short fiber suspensions. The above research has focused mainly in shear flow, however, few articles studied extensional flow with fiber suspensions, especially complex flow.

The objective in this article is to simulate orientation distribution of fiber suspensions based on shear-equibiaxial extensional flow.

\section{MATHEMATICAL MODEL}

The evolution equation of $P$ for a single fiber immersed in a Newtonian flow without external torque was firsty developed by Jeffery[6], which can be expressed as follows:

$$
\dot{P}=W \cdot P+\lambda(E \cdot P-E: P P P) .
$$

(1)Where, $W$ is vorticity tensor of fiber, $E$ is deformation rate of fiber, $\lambda=\left(\beta^{2}-1\right) /\left(\beta^{2}+1\right)$ is the fiber shape factor, $\beta=L / d$ is the fiber aspect ratio. Assuming the fiber internal influence is neglected and the fiber has free orientation in initial conditions[7]. Three dimensional orientation equation can be simplified in this condition:

$$
\psi(P, t)=\frac{1}{4 \pi}\left(\Delta^{T} \cdot \Delta: P P\right)^{-\frac{3}{2}} .
$$

(2)The velocity gradient tensor $\nabla v$, vorticity tensor $W$ and rate of deformation tensor of fiber $E$ can be written as:

$$
\begin{gathered}
\nabla v=\left(\begin{array}{ccc}
\dot{\varepsilon} & \dot{\gamma} & 0 \\
\dot{\gamma} & -2 \dot{\varepsilon} & 0 \\
0 & 0 & \dot{\varepsilon}
\end{array}\right), \\
W=\frac{\nabla v-\nabla v^{T}}{2}=\left(\begin{array}{ccc}
0 & 0 & 0 \\
0 & 0 & 0 \\
0 & 0 & 0
\end{array}\right), \\
E=\frac{\nabla v+\nabla v^{T}}{2}=\left(\begin{array}{ccc}
\dot{\varepsilon} & \dot{\gamma} & 0 \\
\dot{\gamma} & -2 \dot{\varepsilon} & 0 \\
0 & 0 & \dot{\varepsilon}
\end{array}\right) .
\end{gathered}
$$

Where, $v$ is flow velocity, $\dot{\gamma}$ is scalar rate of shear deformation and $\dot{\varepsilon}$ is scalar rate of extensional deformation. Eq.(3c) can be split into two tensor below:

$$
E=\dot{\varepsilon}\left(\begin{array}{ccc}
1 & 0 & 0 \\
0 & -2 & 0 \\
0 & 0 & 1
\end{array}\right)+\dot{\gamma}\left(\begin{array}{ccc}
0 & 1 & 0 \\
1 & 0 & 0 \\
0 & 0 & 0
\end{array}\right)=E^{\prime}+E^{\prime \prime}
$$

(4)Therefore, displacement tensor $\Delta_{i j}$ can be expressed below: 


$$
\frac{\mathrm{d} \Delta_{i j}}{\mathrm{~d} \hat{t}}=-\Delta_{i k}^{\prime} E_{k j}^{\prime}-\Delta_{i k}^{\prime \prime} E_{k j}^{\prime \prime} .
$$

(5)Through Eq.(5) can be shown:

$$
\begin{aligned}
\frac{\mathrm{d} \Delta_{11}^{\prime}}{\mathrm{d} \hat{t}} & =-\left(\Delta_{11}^{\prime} E_{11}+\Delta_{12}^{\prime} E_{21}+\Delta_{31}^{\prime} E_{31}\right)=-\Delta_{11}^{\prime}, \\
\Delta_{11}^{\prime} & =e^{-t} .
\end{aligned}
$$

The other components of $\Delta^{\prime}$ can be acquired by the above similar method. Therefore, deformation tensor $\Delta^{\prime}$ can be shown below:

$$
\Delta^{\prime}=\left(\begin{array}{ccc}
e^{-t} & 0 & 0 \\
0 & e^{2 t} & 0 \\
0 & 0 & e^{-t}
\end{array}\right)
$$

(7)Similarly, Through Eq.(7) can be also shown:

(8)

$$
\frac{\mathrm{d} \Delta_{11}^{\prime \prime}}{\mathrm{d} \hat{t}}=-\left(\Delta_{11}^{\prime \prime} E_{11}^{\prime \prime}+\Delta_{12}^{\prime \prime} E_{21}^{\prime \prime}+\Delta_{31}^{\prime \prime} E_{31}^{\prime \prime}\right)=0, \quad \Delta_{11}^{\prime \prime}=1 .
$$

$\frac{\mathrm{d} \Delta_{12}^{\prime \prime}}{\mathrm{d} \hat{t}}=-\left(\Delta_{11}^{\prime \prime} E_{12}^{\prime \prime}+\Delta_{12}^{\prime \prime} E_{22}^{\prime \prime}+\Delta_{13}^{\prime \prime} E_{32}^{\prime \prime}\right)=-\dot{\gamma}, \quad \Delta_{12}^{\prime \prime}=-\dot{\gamma} T$.

(9)

Therefore:

$$
\Delta^{T} \Delta=\left(\begin{array}{ccc}
0.64 e^{-2 t}+0.32 e^{-t}+0.04 t^{2}+0.04 & -0.16 t e^{2 t}-0.16 t e^{-t}-0.08 t & 0 \\
-0.16 t e^{2 t}-0.16 t e^{-t}-0.08 t & 0.64 e^{4 t}+0.32 e^{2 t}+0.04 t^{2}+0.04 & 0 \\
0 & 0 & 0.64 e^{-2 t}+0.32 e^{-t}+0.04
\end{array}\right) .
$$

Eq.(11) are substituted into Eq.(2) and shown below:

$$
\begin{aligned}
\psi(P, t)= & \frac{1}{4 \pi}\left(\Delta^{T} \cdot \Delta: P P\right)^{-\frac{3}{2}}=\frac{1}{4 \pi}\left[\left(\Delta_{i k}^{T} \Delta_{k j} e_{i} e_{j}\right): P_{s} e_{s} P_{t} e_{t}\right]^{-\frac{3}{2}}=\frac{1}{4 \pi}\left(\Delta_{s k}^{T} \Delta_{k s} P_{s} P_{t}\right)^{-\frac{3}{2}} \\
= & \frac{1}{4 \pi}\left(\Delta_{11}^{T} \Delta_{11} P_{1} P_{1}+\Delta_{22}^{T} P_{2} P_{2}+\Delta_{33}^{T} \Delta_{33} P_{3} P_{3}+\Delta_{12}^{T} \Delta_{12} P_{1} P_{2}+\Delta_{21}^{T} \Delta_{21} P_{2} P_{1}\right)^{-\frac{3}{2}} \\
= & \frac{1}{4 \pi}\left[\left(0.64 e^{-2 t}+0.32 e^{-t}+0.04 t^{2}+0.04\right) \sin ^{2} \theta \cos ^{2} \phi\right. \\
& +\left(0.64 e^{4 t}+0.32 e^{2 t}+0.04 t^{2}+0.04\right) \sin ^{2} \theta \sin ^{2} \phi+\left(0.64 e^{-2 t}+0.32 e^{-t}+0.04\right) \cos ^{2} \theta \\
& \left.+2\left(-0.16 t e^{2 t}-0.16 t e^{-t}-0.08 t\right) \sin ^{2} \theta \sin 2 \phi\right]^{-\frac{3}{2}} .
\end{aligned}
$$

Where, $t=\dot{\gamma} T_{0}$ expresses dimensionless cumulant along with time, $T_{0}$ means current time.

Interval $\theta \in(0, \pi)$ and interval $\phi \in(0,2 \pi)$ are divided 100 nodes respectively. According to Eq.(13) of analytical solution of orientation distribution function of fiber in shear-equibiaxial extensional flow field, several pictures can be shown by numerical method below.

\section{RESULTS AND DISCUSSION}

Figure 1 reflects the evolution process of orientation distribution function of plant fiber in shear-equibiaxial extensional flow field from $T=0.1$ to $T=4.0$. The orientation of plant fiber is clutter at initial moments. In general, there are two peaks. The orientation distribution of plant fiber is wider at the beginning, then it changes narrowed down slowly, i.e., tensile direction is dominant. Where, the orientation of plant fiber is obvious especially at $\theta=\frac{\pi}{2}$. However, it is concentration relatively along 
with the direction of $(\theta, \phi)=\left(\frac{\pi}{2}, 0\right),\left(\frac{\pi}{2}, \pi\right)$, $\left(\frac{\pi}{2}, 2 \pi\right)$. Finally, the basic tending to zero, it can be ignored.

Thus, the maximum value of orientation distribution of plant fiber is the corresponding to a certain value of $\phi$ at $\theta=\frac{\pi}{2}$ in shear-equibiaxial extensional flow field. The maximum value distributions are three locations: $\phi=0, \phi=\pi$ and $\phi=2 \pi$, respectively. Therefore, the position of $\theta=\frac{\pi}{2}$ is fixed, then, the change value of plant fiber orientation along with $\phi$ can be compared.

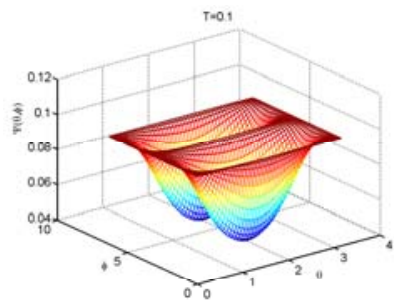

(a)

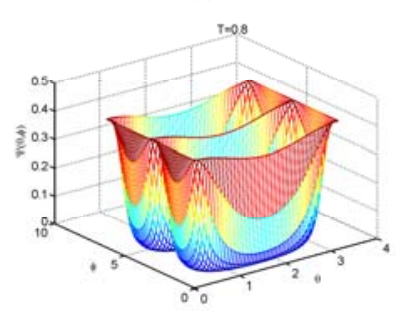

(c)

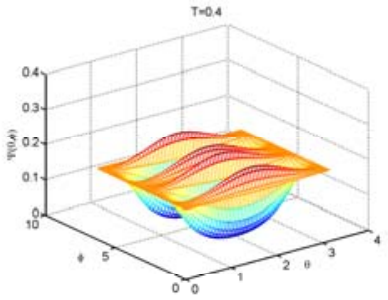

(b)

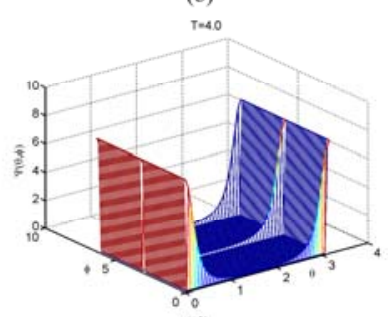

(d)
Fig. 1 The fiber orientation distribution function evolves for shear- equibiaxial extensional flow field

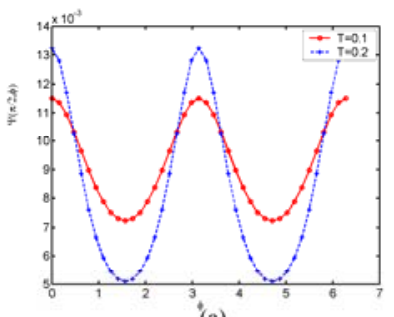

(a)

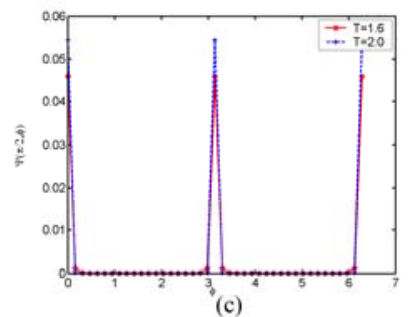

(c)
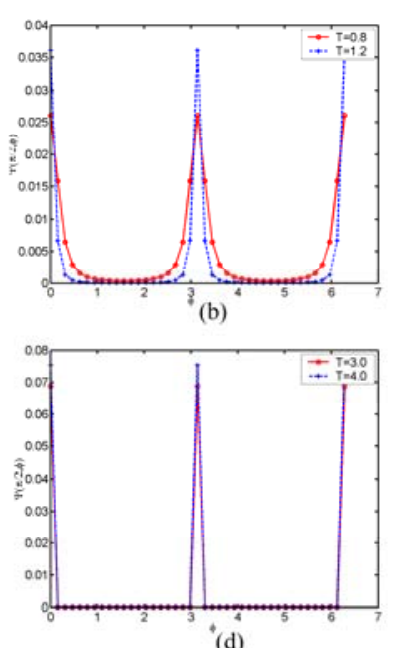

(d)
Fig. 2 The fiber orientation distribution changing for at shear-equibiaxial extensional flow field $\theta=\pi / 2$
From figure 2, it can reflect the orientation change characteristics of plant fiber in the surface $\theta=\frac{\pi}{2}$ clearly. At the beginning, the slope of orientation distribution of plant fiber is slower and wider, it also means that the orientation distribution of plant fiber is relatively clutter. However, the accumulation of extensional deformation is also increased constantly with the increase of time. Furthermore, it can be seen gathered trend at $\phi=0, \pi, 2 \pi$ and the orientation changes obviously increasingly, the degree of orientation is becoming more and more sharp. From Fig. 2 (d), i.e., the orientation of figure at $T=3.0, T=4.0$, it can be seen that the orientation of plant fiber only happens at three directions. In fact, fiber orientation is along with the direction of extend, due to fiber has no difference between head and tail.

\section{CONCLUSIONS}

The greater of tensile deformation rate, the shorter of period of motion; the bigger of length to diameter ratio, the longer of period of motion. The period of motion of short fiber is relevant with extensional flow and length to diameter ratio. The motion of fiber is not the uniform periodic motion, but it is sometimes fast and sometimes slow. The numerical distribution of fiber orientation distribution function has great variation in shear-equibiaxial extensional flow field. When extensional deformation rate is small, its numerical distribution is wide.

\section{ACKNOWLEDGEMENTS}

The authors wish to acknowledge the Guangzhou Huangpu District Project (20140000346), Guangzhou Maritime Institute Talent Introduction Project (A330106) for the financial supports.

\section{REFERENCES}

[1] Parsheh M., Brown M. L., Aidun C. K. International Journal of Multiphase Flow, 2006, 32(12): 1354-1369.

[2] Bay R. S., Tucker C. L. Polymer Composites, 1992, 13:317-321.

[3] Pillai K. M., Tucker C. I., Phelan F. R. Composites Part A: applied science and manufacturing, 2001, (32): 207-220.

[4] Lin J. Z., Liang X. Y., Zhang S. L. Chemical Engineering Research and Design, 2012, 90(6):766-775.

[5] Najam ul Qadir, David A. Jack. Composites: Part A, 2009, 40, 1524-1533.

[6] Jeffery G. B. Proceedings of Royal Society of London,1922,102(715): 161-179.

[7] Bees M. A., Hill N. A., Pedley T. J. Mathematical Biology, 1998, 36(3):269-298. 